\title{
OPEN TMEM150B is dispensable for oocyte maturation and female fertility in mouse
}

Ran Livi ${ }^{1,2,3,4,5}$, Hanni Ke ${ }^{1,2,3,4,5}$, Tong Shao ${ }^{1,2,3,4,5}$, Yingying Oin ${ }^{1,2,3,4,5}$ \& Shidou Zhao ${ }^{1,2,3,4,5 凶}$

Premature ovarian insufficiency (POI) refers to severe decline of ovary function in females which usually leads to infertility. It has been reported that the TMEM150B gene is mostly associated with age at natural menopause, early menopause and POI, but its role in female reproduction remains unknown. In this study, we found Tmem150b was highly expressed in mouse oocytes, but its deletion had no obvious effect on meiotic maturation of oocytes indicated by first polar body emission and spindle morphology. There were also no obvious differences in follicle development and corpus luteum formation between knockout and wild type mice. Finally, knockout of Tmem150b did not affect female fertility and sexual hormone levels. In summary, our results suggest that TMEM150B is not essential for female fertility in mice.

Premature ovarian insufficiency (POI) causes fertility decline in women which is characterized by defect of ovarian function with serum follicle-stimulating hormone (FSH) level over $25 \mathrm{IU} / \mathrm{L}$ before the age of 40 years ${ }^{1,2}$. The prevalence of POI has been reported approximately to be $2.8 \%$ in Chinese women ${ }^{3}$. The etiology of POI is complex and highly heterogeneous. Different factors might be involved in POI including iatrogenic factors, genetic abnormalities, autoimmune disorders, idiopathic and environmental factors, while $20-25 \%$ of cases may be explained by genetic defects ${ }^{4}$. Causative genes associated with POI have been identified, such as genes associated with follicular development (FIGLA, FSHR, NOBOX, GDF9 and BMP15), and genes related to meiosis and DNA repair (STAG3, HFM1, MCM8, MCM9 and MSH5) ${ }^{5}$. However, the etiology of majority of POI cases still remains unclear.

Previous studies have shown that some genetic susceptible loci are shared by age at natural menopause (ANM), early menopause and $\mathrm{POI}^{6}$. Accordingly, several genes related to these loci, such as MCM8, MSH5 and $S Y C P 2 L$, have been identified ${ }^{7,8}$. Not only their knockout mice exhibit infertility, ovarian dysgenesis or premature insufficiency ${ }^{9-11}$, but also mutations in these genes were found in POI patients and the causality was confirmed by functional studies ${ }^{12-15}$. Besides, TMEM150B is one of the genes identified to be associated with ANM and early menopause, which is most significantly correlated with POI in human ${ }^{16}$. While TMEM150B was discovered to promote cell viability under glucose deprivation by prompting autophagy ${ }^{17}$, knockout of an essential autophagy-related gene Atg7 in germ cells resulted in ovarian follicle loss and subfertility in female mice $^{18}$. Whether TMEM150B is required for female fertility has not been investigated yet. Therefore, we generated the Tmem $150 \mathrm{~b}$ knockout mouse model to explore the potential role of TMEM150B in female reproduction.

\section{Materials and methods}

Quantitative reverse-transcription PCR (qRT-PCR). To verify the expression of Tmem $150 \mathrm{~b}$ in oocytes and other tissues, qRT-PCR was performed with wild type mouse tissues. Total RNA was extracted according to the manufacturer's protocol of Qiagen RNase mini kit (Invitrogen, USA). The cDNA was obtained by reverse transcription of RNA using PrimeScript reverse transcriptase (Takara, Japan). Real time PCR was performed using SYBR Green Master Mix (Takara, Japan) with specific primers. Gapdh was used as the internal control. Primer sequences are as follows: Tmem150b, F: 5'-TTGCTGCCTGTCATCTTATTTC-3', R: 5'-AGG TTTTGACGCCCCAGT-3'; Gapdh, F: 5'-AGGTCGGTGTGAACGGATTTG-3', R: 5'-TGTAGACCATGTAGT TGAGGTCA-3'.

\footnotetext{
${ }^{1}$ Center for Reproductive Medicine, Cheeloo College of Medicine, Shandong University, Jinan 250012, Shandong, China. ${ }^{2}$ Key Laboratory of Reproductive Endocrinology of Ministry of Education, Shandong University, Jinan 250012, Shandong, China. ${ }^{3}$ Shandong Key Laboratory of Reproductive Medicine, Jinan 250012, Shandong, China. "Shandong Provincial Clinical Research Center for Reproductive Health, Jinan 250012, Shandong, China. ${ }^{5}$ National Research Center for Assisted Reproductive Technology and Reproductive Genetics, Shandong University, 44 Wenhua Xi Road, Jinan 250012, Shandong, China. ${ }^{\circledR}$ email: shidouzhao@sdu.edu.cn
} 
Establishment of Tmem150b knockout mice. Tmem150b knockout (KO) mice were constructed by CRISPR/Cas9 technique (Cyagen, Suzhou, China) with deletion of 989 bp fragment encompassing exons 2-4. Genotyping was performed by PCR from genomic DNA of mouse tails. Validation of gene knockout in mRNA level was done by RT-PCR using cDNA obtained by reverse transcription of mRNA from mouse ovaries and the PCR products were confirmed by Sanger sequencing. The sequences of primers were as follows: wild type (WT) allele, F: 5'-GACTGCTTGGAGATCCAGCT-3', R: 5'-GTGGAGGCAGTCTGACTATC-3'; and delete allele, dF: 5'-CTTTGTGCCCTGGGTACCTC-3', R: 5'-GTGGAGGCAGTCTGACTATC-3'; F': 5'- AGTGGGACCACA AAAGCAGG-3', R': 5'- CCTTTCACCCACCAGGACAG-3'. All PCR products were separated by $1.5 \%(\mathrm{w} / \mathrm{v})$ agarose gel, stained with ethidium bromide (Invitrogen, USA) and detected by Chemidoc MP System (Bio-Rad, USA). All genetically altered mice had a mixed background of ICR and C57BL/6J. WT mice for experiments were purchased from Charles Rive Company. Mice were housed in a temperature-controlled $\left(22 \pm 2{ }^{\circ} \mathrm{C}\right)$ room with a 12/12 h light/dark cycle and free access to water and food. All animal experiments were performed in accordance to the ethical guidelines approved by Animal Care and Research Committee of Shandong University.

Collection of oocytes. For collection of fully-grown GV stage oocytes, 21-day-old female mice were injected with 5 IU PMSG (Ningbo Sansheng Pharmaceutical Corporation, Zhejiang, China) through intraperitoneal injection. After $46 \mathrm{~h}$, the ovaries were minced with dissection blades before oocytes were collected after 3 times of wash. For MII oocytes collection, mice were super-stimulated with 5 IU PMSG for 46 h and MII stage oocytes were collected after 16 h of hCG (5 IU, Ningbo Sansheng Pharmaceutical Corporation, Zhejiang, China) administration by puncturing the oviduct under light microscopy.

Immunofluorescence. Immunostaining of oocytes was performed as described previously ${ }^{19}$. Briefly, MII oocytes were fixed in $4 \%$ paraformaldehyde $(\mathrm{pH}=7.4)$ for $30 \mathrm{~min}$ at room temperature, permeabilized in $0.3 \%$ TritonX-100 for 20 min, blocked with 1\% BSA dissolved in PBS and then incubated with anti- $\alpha$-Tubulin-FITC antibody for $1 \mathrm{~h}$. DNA was counterstained with propidium iodide for $10 \mathrm{~min}$ at room temperature. Oocytes were mounted on glass slides after being washed three times with PBS and detected for fluorescence under high speed confocal laser scanning microscope (Dragonfly, Andor, England).

Estrous cycle examination. Stages of estrous cycle were detected by preparing vaginal smears in early morning of 2-month-old mice for a period of 14 days. Cells were washed out of vagina with $20 \mu \mathrm{L}$ PBS to glass slide and fixed in 95\% methanol. Vaginal smears were examined after hematoxylin and eosin (H\&E) staining and observed under light microscopy (BX53, Olympus, Japan).

Determination of hormonal profile and histological analysis. To detect sex hormone levels from serum, blood was collected through ophthalmectomy immediately after the general anesthetic. According to standard protocol, serum samples were separated by centrifuging at $4{ }^{\circ} \mathrm{C}, 3000 \times g$ for $30 \mathrm{~min}$. Follicle-stimulating hormone $(\mathrm{FSH})$ and estradiol $\left(\mathrm{E}_{2}\right)$ concentrations were measured by radioimmunoassay (Beijing North Institute of Biotechnology, China).

Ovaries from 3-month and 8-month-old mice were collected and fixed in Bouin's solution at $4^{\circ} \mathrm{C}$ overnight before being transferred to $70 \%$ ( $\mathrm{vol} / \mathrm{vol})$ alcohol. Ovaries were processed, embedded in paraffin with standard protocols and serially sectioned at $5 \mu \mathrm{m}$. Following deparaffinization, ovarian sections were stained with $\mathrm{H} \& \mathrm{E}$ for further histological analysis.

Fertility test. For fertility test, 2-month-old Tmem $150 b^{+/+}$and $T m e m 150 b^{-/-}$females were mated with WT males over a period of 6 months. The number of pups was counted at the day of birth. Fertility was assessed by the total number of pups per genotype, average number of litters per mouse, average number of pups per litter and time to first litter.

Statistical analysis. Data were presented as mean \pm SD and analyzed by Student's $t$ test. Statistical analysis was performed with SPSS 16.0 software. Differences were considered to be statistically significant when $P<0.05$.

\section{Results}

Oocyte meiotic maturation and spindle organization. We firstly analyzed Tmem $150 \mathrm{~b}$ expression in a variety of mouse tissues by qRT-PCR. Tmem $150 b$ mRNA level was low in diverse mouse tissues containing spleen, lung, kidney, testis and ovary, while the expression of Tmem $150 b$ was relatively high in oocytes at GV and MII stages (Fig. 1). These results suggested that Tmem $150 \mathrm{~b}$ might play a role in mouse oocytes.

To investigate the potential role of TMEM150B, we generated Tmem150b KO mouse model by deleting exons 2 to 4 (Fig. 2A). While PCR results using primers $(\mathrm{F}+\mathrm{R})$ for wild type allele yielded $1820 \mathrm{bp}$ fragment, knockout allele produced $831 \mathrm{bp}$ fragment. Meanwhile, PCR using another pair of primers $(\mathrm{dF}+\mathrm{R})$ yielded $413 \mathrm{bp}$ and no bands for wild type and knockout alleles, respectively (Fig. 2B). We further carried out RT-PCR to validate the disruption of the expression of Tmem $150 \mathrm{~b}$ in ovaries from adult mice (Fig. 2C). While the WT allele yielded $524 \mathrm{bp}$ band, KO allele produced $268 \mathrm{bp}$ band. Sanger sequencing also confirmed the deletion of targeted exons 2 to 4 (Fig. 2D). There were no significant differences in body weight and body length between $\mathrm{Tmem} 150 \mathrm{~b}^{+/+}$ and Tmem $150 b^{-/-}$mice according to phenotypical evaluation. MII stage oocytes were collected from WT and $\mathrm{KO}$ mice after overstimulation to determine the role of TMEM150B in oocyte meiotic maturation. Similar rates of first polar body (PB1) extrusion were observed in both genotypes (Fig. 3), suggesting that TMEM150B is dispensable for oocyte maturation. 


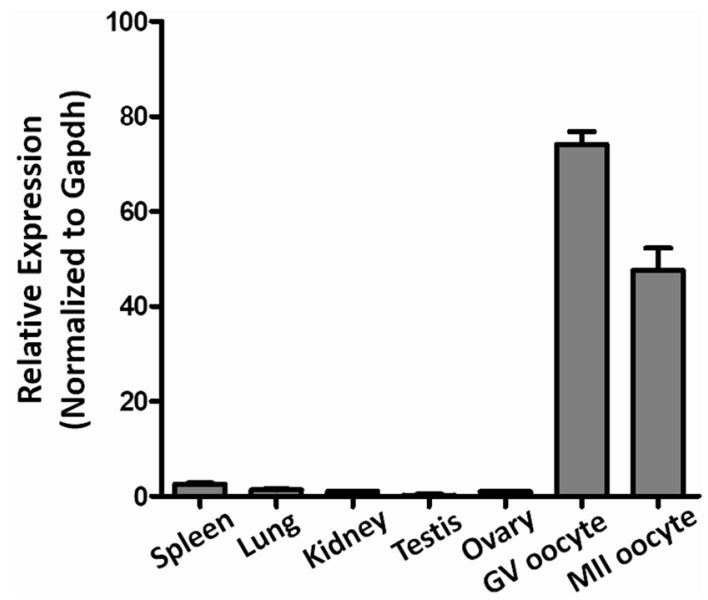

Figure 1. The mRNA expression of Tmem $150 \mathrm{~b}$ in mouse tissues and oocytes detected by qRT-PCR. The expression level of this gene in the ovary was set as " 1 " and other samples were normalized to it.

Next, the spindle morphology of MII stage oocytes was observed from both groups. As shown by immunofluorescence staining, the spindle morphology from Tmem $150 b^{-1-}$ oocyte was similar to wild type oocyte (Fig. 3A). Furthermore, most metaphase oocytes presented typical fusiform-shaped spindles and well-aligned chromosomes at the equatorial plate, suggesting that absence of TMEM150B has no effect on spindle organization.

Estrous cycle and follicular development of adult mice. As there were no changes in oocyte maturation at puberty of mice, we wondered whether TMEM150B deletion had any effect on adult female mice. We examined the estrous cycle through vaginal smear with H\&E staining every morning for 2 weeks. All the Tmem $150 b^{-/-}$mice have regular estrous cycles and normal cell morphology compared with control mice, suggesting that loss of TMEM150B had no obvious effect on estrous cycle (Fig. 4A).

We also observed normal morphology of follicles at different developmental stages from both groups, including primordial follicles, primary follicles, secondary follicles, antral follicles and corpus luteum (Fig. 4B). Granulosa cells were also arranged regularly around oocytes. Collectively, these results suggest that Tmem $150 \mathrm{~b}$ is dispensable for folliculogenesis.

Hormonal profile and female fertility. Fertility test was then performed by mating Tmem $150 b^{+/+}$and Tmem $150 b^{-1-}$ females with WT males for a period of 6 months. Interestingly, all of the adult Tmem $150 b^{-/-}$mice were showed normal fecundity during this time duration. As shown in Table1, the total number of pups in Tmem $150 b^{-/}$group was similar to that in control group. In addition, the average numbers of pups per mouse or per litter were also comparable between the two genotypes. Neither the giving birth at the first time showed any differences. All the newborn pups showed similar size between both genotypes.

In addition, we determined whether Tmem $150 \mathrm{~b}$ deletion causes any change in hormonal profile and ovarian morphology in mice aged 8 months. The levels of FSH and $\mathrm{E}_{2}$ exhibited no obvious alterations between Tmem $150 b^{+/+}$and Tmem $150 b^{-/}$mice (Fig. 5A). Finally, ovaries from both genotypes showed similar amount of follicles from different stages and corpus lutea (Fig. 5B), suggesting that the Tmem $150 b^{-/}$mice also own regular ovulatory ability.

\section{Discussion}

Women suffering from POI face infertility with adverse health outcomes ${ }^{1}$. Despite the etiology of POI has been discovered over decades, the mechanisms still have not been fully investigated. It has been reported that early menopause shares a similar genetic pattern with idiopathic $\mathrm{POI}^{20}$. TMEM150B has been recognized as one of candidate genes which are significantly associated with ANM and early menopause $\mathrm{e}^{7,8}$. Moreover, the SNP rs11668344 located in intron 2 of the TMEM150B gene has been verified to be most significantly associated with POI in European ancestry through genome-wide association study ${ }^{16}$. However, this variant was not verified and no mutation of TMEM150B was identified in the Chinese POI population in our previous study ${ }^{21}$.

It has been reported that the TMEM150B gene was expressed in human ovary ${ }^{17}$. In the present study, we further confirmed the high expression of Tmem $150 b$ in mouse oocytes in immature and mature stages. The deletion of genomic DNA from our gene-edited mice was large enough to truncate the TMEM150B protein and make it non-functional. Consequently, we found that the deletion of Tmem $150 \mathrm{~b}$ had no effect on follicle maturation and oocyte development, as indicated by similar PB1 extrusion rate and MII oocyte morphology. Furthermore, 


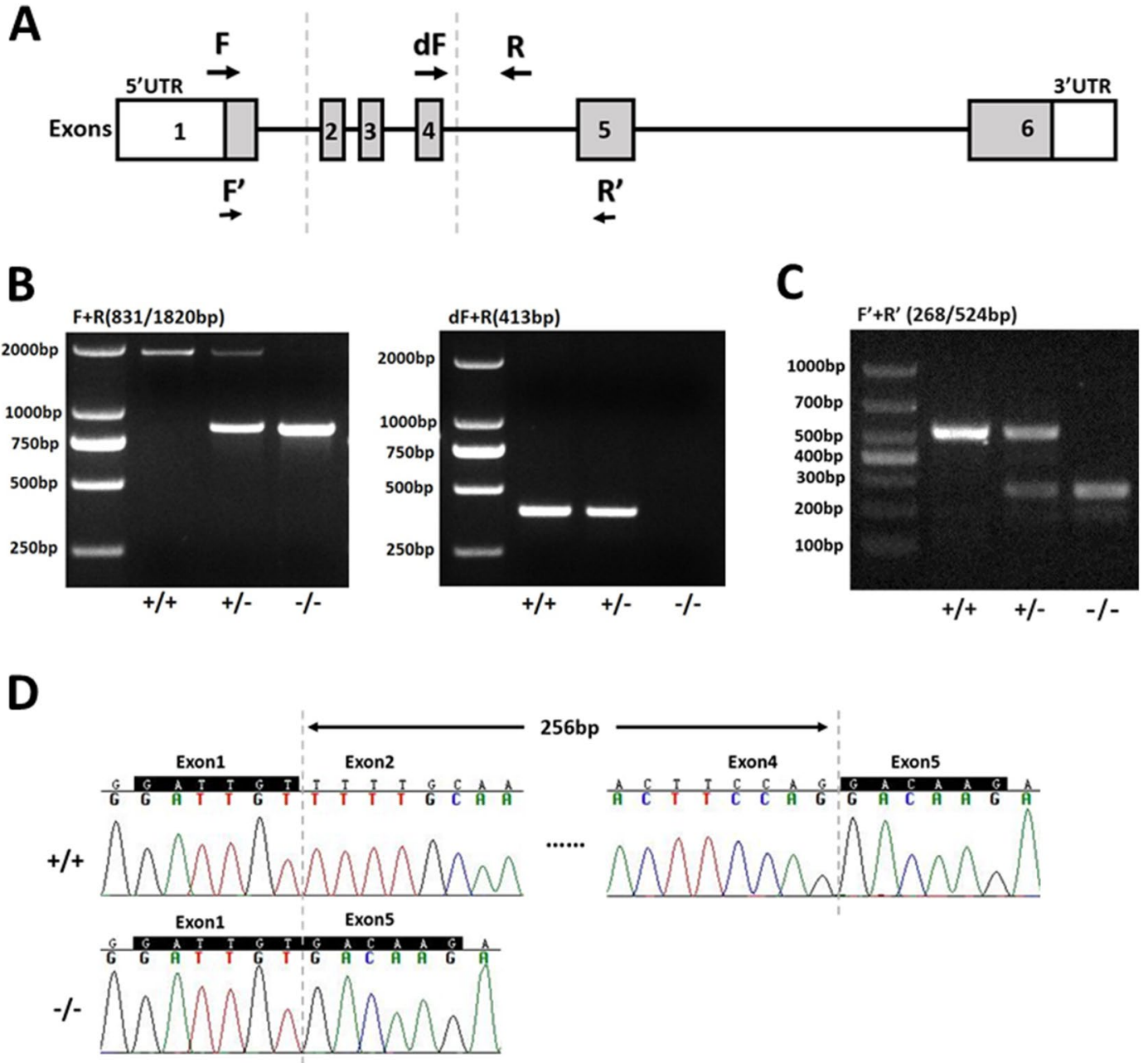

Figure 2. Targeted disruption of the Tmem $150 b$ in mouse genomic DNA and mRNA. (A) Schematic representation of the mouse Tmem $150 \mathrm{~b}$ gene. The exons and introns deleted are indicated by dashed line. Arrows show positions of PCR primers used for genotyping in genomic DNA and confirming the disruption of gene in mRNA level. (B) Genotyping of mice by PCR amplification. The PCR using primers (F+R) for wild type and knockout alleles yielded $1820 \mathrm{bp}$ and $831 \mathrm{bp}$ fragments respectively. The PCR using primers $(\mathrm{dF}+\mathrm{R})$ for wild type and knockout alleles yielded $413 \mathrm{bp}$ and no bands respectively. (C) Confirming the knockout of Tmem $150 b$ in mRNA using RT-PCR with the primers (F' + R') for wild type and knockout alleles yielding 524 bp and $268 \mathrm{bp}$ fragments, respectively. (D) Sanger sequencing showed the deletion of exons 2 to 4 in Tmem150b mRNA from Tmem $150 b^{---}$mouse ovary.

fertility test showed similar number of pups and litter size in both genotypes. Estrous cycle and hormonal profile also exhibited no significant differences between both genotypes. Our findings suggest that TMEM150B is dispensable for folliculogenesis, oocyte maturation and female fertility in mice.

Although our study demonstrates that Tmem $150 \mathrm{~b}$ does not play a prominent role in follicle development and fertility of female mice, there may be some limitations. TMEM150B, also named as DRAM-related/associated member 3 (DRAM-3), is one of the five members of the DRAM family which includes DRAM-1, DRAM-2 and TMEM150A/B/ $\mathrm{C}^{17,22}$ and functional redundancy cannot be excluded. It may be better to mutate all interrelated genes to eliminate the compensation from paralogs. Besides, we only detected the phenotype at basal status in normal condition, and could not exclude the occurrence of POI phenotype under stress conditions.

In summary, we demonstrate that the female Tmem $150 b^{-/}$mice have normal fertility. Together with our previous study identifying no causative variants in POI patients, it is suggested that the TMEM150B gene may not be a POI causative gene. Nevertheless, our study may help the scientific community reveal the etiology of POI. 
A
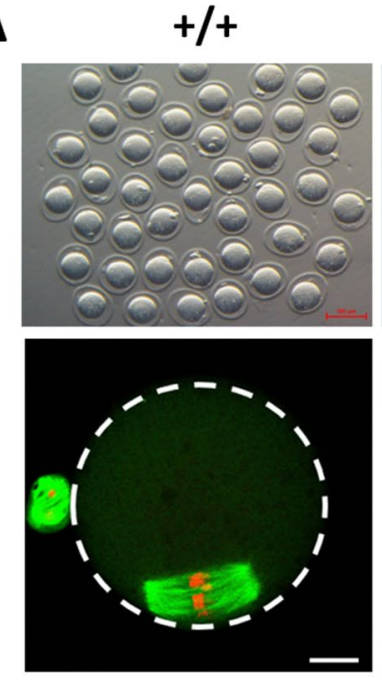

$-/-$
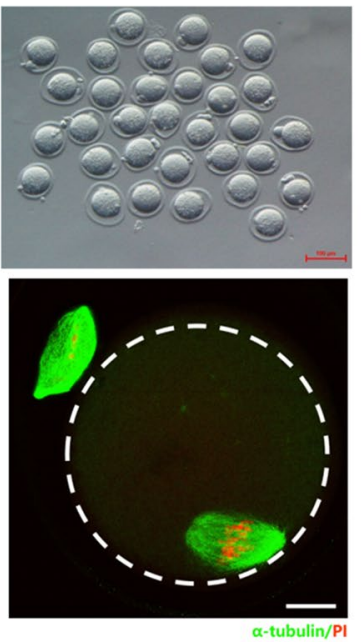

B

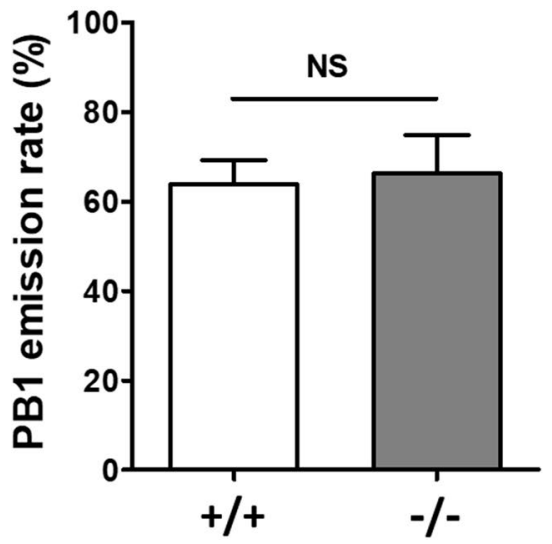

Figure 3. Oocyte maturation analysis by morphology and immunofluorescence staining. (A) Representative morphology of MII stage oocytes after superovulation at postnatal day 21 derived from Tmem $150 b^{+/+}$and Tmem $150 b^{-/-}$mice. Scale bar $=100 \mu \mathrm{m}$. The $\alpha$-tubulin was stained by a fluorescein isothiocyanate (FITC)conjugated antibody, and DNA by PI. Scale bar $=10 \mu \mathrm{m}$. (B) Statistical analysis of PB1 emission rates between Tmem150b ${ }^{+/+}(\mathrm{n}=236)$ and Tmem150b ${ }^{-/-}(\mathrm{n}=230)$ MII oocytes. NS, no significance.
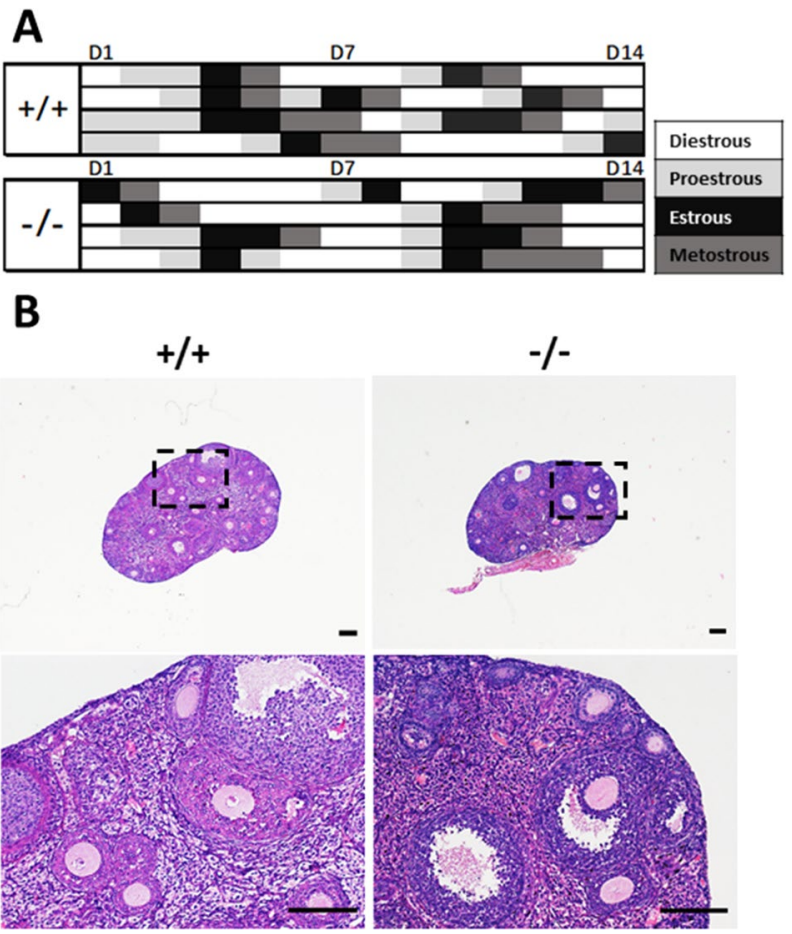

Figure 4. Estrous cycle analysis of female mice and morphology analysis of ovaries by H\&E staining. (A) Regular estrous cycle of 2-month-old female mice from both genotypes. (B) Ovarian histological analysis of 3-month-old Tmem150b ${ }^{+/+}$and Tmem $150 b^{-/-}$females by H\&E staining. Scale bar $=100 \mu \mathrm{m}$. 


\begin{tabular}{|c|l|l|l|l|}
\hline Genotypes & Total pups & Litters/Mouse & Pups/Litter & $\begin{array}{l}\text { Time to first litter } \\
\text { (days) }\end{array}$ \\
\hline$+/+(\mathrm{n}=5)$ & 177 & $4.0 \pm 0.45$ & $8.85 \pm 1.53$ & $95 \pm 3.98$ \\
\hline$-/-(\mathrm{n}=5)$ & 189 & $4.2 \pm 0.37$ & $8.59 \pm 0.82$ & $98 \pm 6.18$ \\
\hline
\end{tabular}

Table 1. Fertility test results of two mouse genotypes.

A

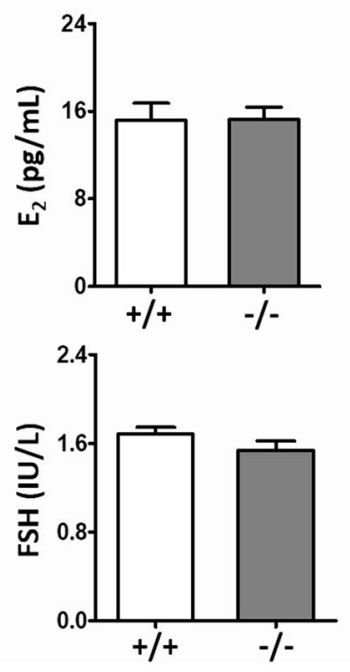

B

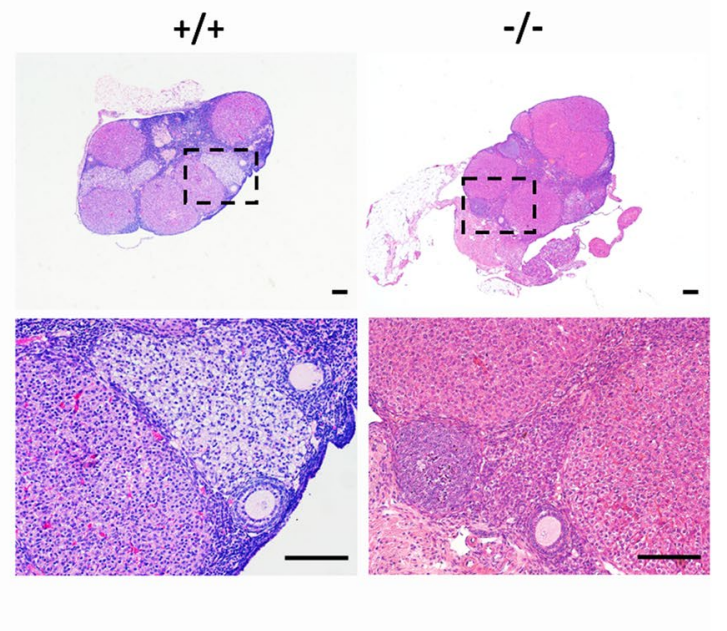

Figure 5. Hormone and ovary morphology analyses after fertility test. (A) No significant changes in FSH and $\mathrm{E}_{2}$ concentrations in serum of 8-month-old mice between $\mathrm{Tmem}_{150 b^{++}}(\mathrm{n}=5)$ and $\mathrm{Tmem} 150 b^{--}(\mathrm{n}=5)$ group. (B) Ovarian histological analysis with H\&E staining after fertility test from both genotypes. Scale bar $=100 \mu \mathrm{m}$.

Received: 4 August 2020; Accepted: 25 November 2020

Published online: 07 December 2020

\section{References}

1. De Vos, M., Devroey, P. \& Fauser, B. C. J. M. Primary ovarian insufficiency. Lancet 376, 911-921 (2010).

2. Lawrence, M. \& Nelsom, M. D. Primary ovarian insufficiency. N. Engl. J. Med. 360, 606-614 (2009).

3. Wu, X. et al. Impact of premature ovarian failure on mortality and morbidity among Chinese women. PLoS ONE 9(3), e89597 (2014).

4. Qin, Y., Jiao, X., Simpson, J. L. \& Chen, Z. J. Genetics of primary ovarian insufficiency: new developments and opportunities. Hum. Reprod. Update 21, 87-808 (2015).

5. Jiao, X., Ke, H., Qin, Y. \& Chen, Z. J. Molecular genetics of premature ovarian insufficiency. Trends Endocrinol. Metab. 29, 795-807 (2018).

6. Laisk-Podar, T. et al. Ovarian physiology and GWAS: biobanks, biology, and beyond. Trends Endocrinol. Metab. 27, 516-528 (2016).

7. Stolk, L. et al. Meta-analyses identify 13 loci associated with age at menopause and highlight DNA repair and immune pathways. Nat. Genet. 44, 260-268 (2012).

8. Day, F. R. et al. Large-scale genomic analyses link reproductive aging to hypothalamic signaling, breast cancer susceptibility and BRCA1-mediated DNA repair. Nat. Genet. 47, 1294-1303 (2015).

9. Lutzmann, M. et al. MCM8- and MCM9-deficient mice reveal gametogenesis defects and genome instability due to impaired homologous recombination. Mol. Cell 47, 523-534 (2012).

10. Edelmann, W. et al. Mammalian MutS homologue 5 is required for chromosome pairing in meiosis. Nat. Genet. 21, 123-127 (1999).

11. Zhou, J. et al. Accelerated reproductive aging in females lacking a novel centromere protein SYCP2L. Hum. Mol. Genet. 24, 6505-6514 (2015).

12. AlAsiri, S. et al. Exome sequencing reveals MCM8 mutation underlies ovarian failure and chromosomal instability. J. Clin. Invest. 125, 258-262 (2015).

13. Dou, X. et al. Minichromosome maintenance complex component 8 mutations cause primary ovarian insufficiency. Fertil. Steril. 106, 1485-1489 (2016).

14. Guo, T. et al. Mutations in MSH5 in primary ovarian insufficiency. Hum. Mol. Genet. 26, 1452-1457 (2017).

15. He, W.B. et al. Homozygous variants in SYCP2L cause premature ovarian insufficiency. J. Med. Genet. 1-5 (2020).

16. Perry, J. R. et al. A genome-wide association study of early menopause and the combined impact of identified variants. Hum. Mol. Genet. 22, 1465-1472 (2013).

17. Mrschtik, M. et al. DRAM-3 modulates autophagy and promotes cell survival in the absence of glucose. Cell Death Differ. 22, 1714-1726 (2015).

18. Song, Z. H. et al. Germ cell-specific Atg7 knockout results in primary ovarian insufficiency in female mice. Cell Death Dis. 15, e1589 (2015).

19. Zeng, J. et al. SIRT4 is essential for metabolic control and meiotic structure during mouse oocyte maturation. Aging Cell 17, e12789 (2018). 
20. Tibiletti, M. G. et al. The idiopathic forms of premature menopause and early menopause show the same genetic pattern. Hum. Reprod. 14, 2731-2734 (1999).

21. Shao, T., Ke, H., Liu, R., Zhao, S. \& Qin, Y. Variation analysis of the TMEM150B gene in Chinese women with premature ovarian insufficiency. Reprod. Biomed. Online 38, 407-412 (2019).

22. Mrschtik, M. \& Ryan, K. M. Another DRAM involved in autophagy and cell death. Autophagy 12, 603-605 (2016).

\section{Author contributions}

S.Z. and Y.Q. conceived the idea, supervised the experiments, and revised the manuscript. R.L wrote the main manuscript text and prepared Figs. 1,2,4 and 5. H.K provided Fig. 3. T.S provided the support in feeding animals. All authors reviewed the manuscript.

\section{Funding}

This study was supported by grants from the National Key Research \& Developmental Program of China (2017YFC1001100 and 2018YFC1003800), National Natural Science Foundation of China (81873823 and 81771541), Shandong Province Natural Science Foundation for Distinguished Youth (JQ201720), Young Scholars Program of Shandong University (2016WLJH26) and The Fundamental Research Funds of Shandong University.

\section{Competing interests}

The authors declare no competing interests.

\section{Additional information}

Correspondence and requests for materials should be addressed to S.Z.

Reprints and permissions information is available at www.nature.com/reprints.

Publisher's note Springer Nature remains neutral with regard to jurisdictional claims in published maps and institutional affiliations.

Open Access This article is licensed under a Creative Commons Attribution 4.0 International License, which permits use, sharing, adaptation, distribution and reproduction in any medium or format, as long as you give appropriate credit to the original author(s) and the source, provide a link to the Creative Commons licence, and indicate if changes were made. The images or other third party material in this article are included in the article's Creative Commons licence, unless indicated otherwise in a credit line to the material. If material is not included in the article's Creative Commons licence and your intended use is not permitted by statutory regulation or exceeds the permitted use, you will need to obtain permission directly from the copyright holder. To view a copy of this licence, visit http://creativecommons.org/licenses/by/4.0/.

(C) The Author(s) 2020 